\title{
Coronary computed tomography angiography: How should we act on what we find?
}

\author{
Márcio Sommer Bittencourt, $\mathrm{MD}, \mathrm{MPH}, \mathrm{PhD},{ }^{\mathrm{a}, \mathrm{b}}$ and Ron Blankstein, $\mathrm{MD}^{\mathrm{c}}$ \\ ${ }^{a}$ Center for Clinical and Epidemiological Research, University Hospital and State of São Paulo \\ Cancer Institute (ICESP), University of São Paulo, São Paulo, Brazil \\ ${ }^{b}$ Preventive Medicine Center, Hospital Israelita Albert Einstein, São Paulo, Brazil \\ c Non-Invasive Cardiovascular Imaging Program, Departments of Medicine (Cardiovascular \\ Division) and Radiology, Brigham and Women's Hospital and Harvard Medical School, Boston, \\ MA
}

Received Mar 21, 2016; accepted Mar 29, 2016

doi: 10.1007/s12350-016-0494-3

\section{See related article, pp. $1267-1278$}

Coronary computed tomography angiography (CTA) has progressed from an experimental imaging technique to a commonly performed clinical exam in less than 20 years. This rapid evolution was enabled by studies documenting excellent diagnostic accuracy ${ }^{1,2}$ as well as the prognostic value of detecting non-obstructive and obstructive coronary artery disease (CAD). ${ }^{3-5}$ Recent clinical trials have shown the utility of coronary CTA among patients presenting to the emergency department with acute chest pain ${ }^{6}$ and among those with stable symptoms in the outpatient setting. ${ }^{7-9}$ In the present issue of JNC, a study by Uretsky et al has expanded this list by evaluating the use of CTA among individuals admitted to hospital with acute chest pain and unremarkable troponins and electrocardiographic findings. ${ }^{10}$

Despite differences in clinical setting, several common findings have been observed among studies comparing coronary CTA to functional testing. First, both strategies appear to be safe and effective for the evaluation of patients with suspected CAD. Second, the rate of downstream adverse events is low, regardless of how patients are evaluated (a finding which is in part

Reprint requests: Ron Blankstein, MD, Non-Invasive Cardiovascular Imaging Program, Departments of Medicine (Cardiovascular Division) and Radiology, Brigham and Women's Hospital and Harvard Medical School, 75 Francis St, Boston, MA 02115; rblankstein@partners.org

J Nucl Cardiol 2017;24:1279-81.

1071-3581/\$34.00

Copyright (c) 2016 American Society of Nuclear Cardiology. due to over-estimation of risk among patients referred for non-invasive imaging in contemporary practice). Finally, the rate of invasive coronary angiography (ICA) and coronary revascularization is higher among patients randomized to coronary CTA. Since the increase in these procedures is not associated with a meaningful reduction in events, the ideal strategy to evaluate individual with chest pain of suspected cardiac origin remains a topic of intense debate.

It is important to note that most studies were designed to evaluate the clinical effectiveness of using coronary CTA and did not dictate how the results of this test should be used in patient management decisions (with the exception of two trials which suggested ICA if a stenosis $\geq 70 \%$ was detected). ${ }^{11,12}$ Therefore, the need and choice of further therapies and procedures was left to treating physicians. This was also the case for the current study by Uretsky et al which made several noteworthy observations. First, following hospital admission, there was an overall increase in the use of medical therapy, including aspirin, statin, and anti-hypertensive/anti-ischemic drugs. However, the increase in medication use was similar in the two groups despite significant differences in the rate of abnormal test results. While only $5 \%$ of the stress tests were abnormal, the absolute increase in the use of aspirin and statins was $24 \%$ and $18 \%$, respectively. For coronary CTA, the prevalence of obstructive CAD $(\geq 50 \%)$ was $11 \%$, while the absolute increase in the use of aspirin and statins was $16 \%$ and $17 \%$, respectively. Although in some cases this increase in medication use on the CTA arm may have been driven by the detection of non-obstructive disease, a similar increase in both groups suggests that physicians relied on data other than imaging findings to decide on the need for medical therapies. 
These findings by Uretsky et al contrast with the recently presented results of the PROMISE trial, ${ }^{13}$ where the use of coronary CTA led to higher initiation of aspirin $(11.8 \%$ vs $7.8 \%, P<.001)$, statin $(12.7 \%$ vs $6.2 \%, P<.001)$, and beta-blockers $(8.1 \%$ vs $5.3 \%$, $P<.001$ ). Similarly, in the SCOT-HEART study, the use of coronary CTA led to higher use of preventive and anti-anginal treatments. ${ }^{8}$ While this significant increase may have been driven by the detection of non-obstructive plaque, the explanation for these findings remains speculative until data regarding medication changes are presented according to test findings. Nevertheless, two prior studies have evaluated the use of preventive medical therapies stratified by coronary CTA in routine clinical care. ${ }^{14,15}$ Both showed an increase in aspirin and statin that was proportional to the severity of CAD detected by CTA. However, the use of aspirin and statins also increased in the group with no plaque on coronary CTA, suggesting that other clinical factors may influence physician management decisions.

On the other hand, data on appropriate withdrawal of medications based on testing are far less studied. While de-intensification of treatment is not proposed by any guidelines, many patients may be stratified to a much lower risk group by the new test findings (e.g., normal coronary CTA). In such a scenario, the use of aspirin may result in more harm than benefit, ${ }^{16}$ and the statin dose may be decreased (or in some cases discontinued) to match the lower risk of future events. No data on the rate of reduction of medication use were provided by either Uretsky et al or by the PROMISE study. The only data on de-intensification of therapy after coronary CTA suggest that a normal coronary CTA is associated with de-intensification of statin dose in $18 \%$ of individuals, and with aspirin cessation in $5 \% .{ }^{14}$ Other studies have also suggested that physicians are much better at intensifying treatment than appropriately reducing it. ${ }^{17,18}$ A recently published study of Veterans Health Administration demonstrated that only a minority of cases had their hypertension or diabetes medication de-intensified despite adequate achievement of targets and older age. ${ }^{18}$ Due to the low rate of events in the studies of chest pain evaluated by coronary CTA and the even lower rates of events in individuals with normal coronary CTA, the appropriateness of de-intensification of treatment needs to be further evaluated. Nevertheless, since, regardless of treatment, the event rates in such groups are extremely low, any studies attempting to find a difference in outcomes will be extremely challenging to conduct.

The second aspect of the treatment decision after a non-invasive test in the evaluation of chest pain of suspected cardiac origin is the increase in downstream ICA and coronary revascularizations. In keeping with prior studies, Uretsky et al also observed a significant increase in ICA ( $2 \%$ vs $11 \%, P=.001)$ and coronary revascularizations ( $1 \%$ vs $7 \%, P=.002)$. Yet, in $36 \%$ (7 of 19) of the patients who underwent an ICA after coronary CTA, the initial CTA identified either normal or non-obstructive $\mathrm{CAD}$, and within this group, 3 individuals underwent coronary revascularization.

Although coronary CTA is performed for diagnostic purposes when used for evaluating chest pain of unknown origin, its findings carry well-validated prognostic information, which can be used for selecting candidates for ICA and coronary revascularization. According to the current guidelines on stable CAD, ICA should only be performed when the results of the noninvasive testing indicate a high likelihood of severe $\mathrm{CAD}$ amenable to coronary revascularizations, or in selected cases of refractory symptoms. ${ }^{19}$ The guidelines define severe CAD on coronary CTA as multivessel disease with $\geq 70 \%$ stenosis or left main stenosis $\geq 50 \%$. Thus, many individuals with $<70 \%$ stenosis and some of the individuals with $\geq 70 \%$ (those with single-vessel disease) may adequately be medically managed after the CAD diagnosis by coronary CTA. Although not currently addressed by the guidelines, another possible strategy would be to further evaluate the future risk of events in selected individuals with moderate or severe CAD detected by CTA using stress testing. Thus, while the diagnosis of CAD and the need for intensive medical therapy (both preventive and anti-anginal medications) could be adequately performed based on the extent and severity of CAD on CTA, the need for ICA or revascularization should be reserved for patients with highrisk anatomy or those that have evidence of ischemia on functional testing.

The current study by Uretsky et al provides information on how physicians acted on the results provided by coronary CTA and stress testing. Among the group randomized to coronary CTA, $14 \%$ underwent additional stress testing during follow-up. Among the 6 individuals with $50-70 \%$ stenosis, most (83\%) were referred to further testing. Specifically, 3 underwent additional stress testing and 3 underwent invasive angiography. Among those with $\geq 70 \%$ stenosis, $12(75 \%)$ required further testing (38\% underwent additional stress testing and 56\% underwent invasive angiography). Interestingly, additional testing also occurred in 8 individuals with normal coronaries $(8 \%)$ or in those with non-obstructive disease $(11 \%)$, indicating that physicians occasionally questioned a "negative" coronary CTA, despite its wellvalidated negative predictive value and excellent prognosis. One other remarkable finding of this study is that physicians did not use coronary CTA following inconclusive stress testing. Among individuals randomized to initial stress testing, $15 \%$ underwent additional non- 
invasive imaging, with most $(11 \%)$ being additional stress testing, and only a minority representing a referral to coronary CTA $(4 \%)$.

Collectively, the results of the current study by Uretsky and prior studies of coronary CTA vs usual care for the evaluation of CAD across other clinical settings suggest that both strategies are acceptable options for the evaluation of chest pain of suspected cardiac origin. When comparing the current results with other studies with similar design, a high variability in the use of medical therapy, additional non-invasive testing, ICA, and coronary revascularizations was noted. This suggests that there are gaps in the knowledge of how to most effectively act upon the results of testing. Thus, in order to make the best use of the results provided by non-invasive imaging tests, further studies should aim to understand the reasons for this variability in downstream patient management decisions. Additionally, future studies are needed comparing different therapeutic strategies for different sub-groups based on imaging findings. Such data could help define the most appropriate way to act on various stress results, and ultimately improve patient outcomes.

\section{References}

1. Miller JM, Rochitte CE, Dewey M, Arbab-Zadeh A, Niinuma H, Gottlieb I, et al Diagnostic performance of coronary angiography by 64-row CT. N Engl J Med 2008;359:2324-36.

2. Budoff MJ, Dowe D, Jollis JG, Gitter M, Sutherland J, Halamert E, et al Diagnostic performance of 64-multidetector row coronary computed tomographic angiography for evaluation of coronary artery stenosis in individuals without known coronary artery disease: Results from the prospective multicenter ACCURACY (Assessment by Coronary Computed Tomographic Angiography of Individuals Undergoing Invasive Coronary Angiography) trial. J Am Coll Cardiol 2008;52:1724-32.

3. Min JK, Shaw LJ, Devereux RB, Okin PM, Weinsaft JW, Russo DJ, et al Prognostic value of multidetector coronary computed tomographic angiography for prediction of all-cause mortality. J Am Coll Cardiol 2007;50:1161-70.

4. Bittencourt MS, Hulten E, Ghoshhajra B, O'Leary D, Christman MP, Montana P, et al Prognostic value of nonobstructive and obstructive coronary artery disease detected by coronary computed tomography angiography to identify cardiovascular events. Circ Cardiovasc Imaging 2014;7:282-91.

5. Chow BJW, Wells GA, Chen L, Yam Y, Galiwango P, Abraham A, et al Prognostic value of 64-slice cardiac computed tomography severity of coronary artery disease, coronary atherosclerosis, and left ventricular ejection fraction. J Am Coll Cardiol 2010;55:1017-28.

6. Hulten E, Pickett C, Bittencourt MS, Villines TC, Petrillo S, Di Carli MF, et al Outcomes after coronary computed tomography angiography in the emergency department: A systematic review and meta-analysis of randomized, controlled trials. J Am Coll Cardiol 2013;61:880-92.

7. Douglas PS, Hoffmann U, Patel MR, Mark DB, Al-Khalidi HR, Cavanaugh B, et al Outcomes of anatomical versus functional testing for coronary artery disease. N Engl J Med 2015;372:1291-300.
8. S-H investigators. CT coronary angiography in patients with suspected angina due to coronary heart disease (SCOT-HEART): An open-label, parallel-group, multicentre trial. Lancet 2015;385:2383-91.

9. McKavanagh P, Lusk L, Ball PA, Verghis RM, Agus AM, Trinick $\mathrm{TR}$, et al A comparison of cardiac computerized tomography and exercise stress electrocardiogram test for the investigation of stable chest pain: The clinical results of the CAPP randomized prospective trial. Eur Heart J Cardiovasc Imaging 2015;16:441-8.

10. Uretsky S, Argulian E, Supariwala A, Agarwal SK, El-Hayek G, Chavez $\mathrm{P}$, et al Comparative effectiveness of coronary CT angiography versus stress cardiac imaging in patients following hospital admission for chest pain Work-up: The ProspEctive First Evaluation in Chest Pain (PERFECT) Trial. J Nucl Cardiol 2016. doi:10.1007/s12350-015-0354-6.

11. Goldstein JA, Chinnaiyan KM, Abidov A, Achenbach S, Berman DS, Hayes SW, et al The CT-STAT (coronary computed tomographic angiography for systematic triage of acute chest pain patients to treatment) trial. J Am Coll Cardiol 2011;58: 1414-22.

12. Goldstein JA, Gallagher MJ, O’Neill WW, Ross MA, O'Neil BJ, Raff GL. A randomized controlled trial of multi-slice coronary computed tomography for evaluation of acute chest pain. J Am Coll Cardiol 2007;49:863-71.

13. Ladapo JA, Hoffmann U, Lee KL, Coles A, Huang M, Mark DB, et al Abstract 14051: Changes in Medical Therapy and Lifestyle After Anatomical Versus Functional Testing for Coronary Artery Disease: The PROMISE Trial (PROspective Multicenter Imaging Study for Evaluation of Chest Pain). Circulation 2015;132: A14051.

14. Cheezum MK, Hulten EA, Smith RM, Taylor AJ, Kircher J, Surry $\mathrm{L}$, et al Changes in preventive medical therapies and $\mathrm{CV}$ risk factors after CT angiography. JACC Cardiovasc Imaging 2013;6:574-81.

15. Hulten E, Bittencourt MS, Singh A, O'Leary D, Christman MP, Osmani $\mathrm{W}$, et al Coronary artery disease detected by coronary computed tomographic angiography is associated with intensification of preventive medical therapy and lower low-density lipoprotein cholesterol. Circ Cardiovasc Imaging 2014;7:629-38.

16. Miedema MD, Duprez DA, Misialek JR, Blaha MJ, Nasir K, Silverman MG, et al Use of coronary artery calcium testing to guide aspirin utilization for primary prevention: Estimates from the multi-ethnic study of atherosclerosis. Circ Cardiovasc Qual Outcomes 2014;7:453-60.

17. Nasir K, Bittencourt MS, Blaha MJ, Blankstein R, Agatson AS, Rivera JJ, et al Implications of coronary artery calcium testing among statin candidates according to American College of Cardiology/American Heart Association cholesterol management guidelines MESA (Multi-Ethnic Study of Atherosclerosis). J Am Coll Cardiol 2015;66:1657-68.

18. Sussman JB, Kerr EA, Saini SD, Holleman RG, Klamerus ML, Min LC, et al Rates of deintensification of blood pressure and glycemic medication treatment based on levels of control and life expectancy in older patients with diabetes mellitus. JAMA Intern Med 2015;175:1942-9. doi:10.1001/jamainternmed.2015.5110.

19. Fihn SD, Blankenship JC, Alexander KP, Bittl JA, Byrne JG, Fletcher BJ, et al 2014 ACC/AHA/AATS/PCNA/SCAI/STS focused update of the guideline for the diagnosis and management of patients with stable ischemic heart disease: A report of the American College of Cardiology/American Heart Association Task Force on Practice Guidelines, and the American Association for Thoracic Surgery, Preventive Cardiovascular Nurses Association, Society for Cardiovascular Angiography and Interventions, and Society of Thoracic Surgeons. Circulation 2014;130:1749-67. 